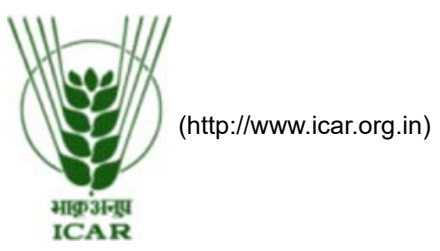

\title{
KRISHI (http://krishi.icar.gov.in)
}

ICAR RESEARCH DATA REPOSITORY FOR KNOWLEDGE MANAGEMENT

(An Institutional Publication and Data Inventory Repository)

\author{
KRISHI Publication and Data Inventory Repository (/jspui/) \\ / Horticultural Science A7 (/jspui/handle/123456789/8) \\ / ICAR-Indian Institute of Horticultural Research K5 (/jspui/handle/123456789/108) \\ / HS-IIHR-Publication (/jspui/handle/123456789/513)
} "Not Available": Please do not remove the default option "Not Available" for the fields where metadata
information is not available

"1001-01-01": Date not available or not applicable for filling metadata infromation

Please use this identifier to cite or link to this item:

http://krishi.icar.gov.in/jspui/handle/123456789/36826

Title:

Other Titles:

Authors:

\section{Plant Resistance to Geminiviruses}

Not Available

Basavaprabhu L. Patil (/jspui/browse?type=author\&value=Basavaprabhu+L.+Patil) Supriya Chakraborty (/jspui/browse?type=author\&value=Supriya+Chakraborty) Henryk Czosnek (/jspui/browse?type=author\&value=Henryk+Czosnek) Elvira Fiallo-Olivé (/jspui/browse?type=author\&value=Elvira+Fiallo-Oliv\%C3\%A9) Robert L. Gilbertson (/jspui/browse?type=author\&value=Robert+L.+Gilbertson) James Legg (/jspui/browse?type=author\&value=James+Legg) Shahid Mansoor (/jspui/browse?type=author\&value=Shahid+Mansoor) Jesús Navas-Castillo (/jspui/browse?type=author\&value $=$ Jes\%C3\%BAs+NavasCastillo)

Rubab Z. Naqvi (/jspui/browse?type=author\&value=Rubab+Z.+Naqvi)

Saleem U. Rahman (/jspui/browse?type=author\&value=Saleem+U.+Rahman) Francisco M. Zerbini (/jspui/browse?type=author\&value=Francisco+M.+Zerbini)

ICAR Data Use http://krishi.icar.gov.in/PDF/ICAR_Data_Use_Licence.pdf (http://krishi.icar.gov.in Licennce: /PDF/ICAR_Data_Use_Licence.pdf)

Author's Affiliated institute:
ICAR-Indian Institute of Horticultural Research Jawaharlal Nehru University, New Delhi, India The Hebrew University of Jerusalem, Rehovot, Israel Institute for Mediterranean and Subtropical Horticulture "La Mayora"-Spanish National Research Council-University of Malaga, Algarrobo-Costa, Málaga, Spain University of California, Davis, CA, United States International Institute of Tropical Agriculture, Dar-Es-Salaam, Tanzania National Institute for Biotechnology and Genetic Engineering, Faisalabad, Pakistan Institute for Mediterranean and Subtropical Horticulture "La Mayora"-Spanish National Research Council-University of Malaga, Algarrobo-Costa, Málaga, Spain National Institute for Biotechnology and Genetic Engineering, Faisalabad, Pakistan Federal University of Viçosa, Viçosa, MG, Brazil

\section{Published/ Complete 2020-06-02}

Date: 